\title{
Optical Imaging and Tissue Characterization with Polarization Discrimination of Time-Gated Signals
}

\author{
Chia-Wei Sun, Yih-Ming WANG, Chih-Chung YANG*, Yean-Woei KIANG, Long-Sheng LU ${ }^{1}$ and Ming-Jai SU ${ }^{1}$ \\ Graduate Institute of Electro-Optical Engineering, Graduate Institute of Communication Engineering \\ and Department of Electrical Engineering, National Taiwan University, 1, Roosevelt Road, Sec. 4, Taipei, Taiwan, R.O.C. \\ ${ }^{1}$ Graduate Institute of Pharmacology, National Taiwan University, Taipei, Taiwan, R.O.C. \\ (Received March 6, 2003; Accepted May 21, 2003)
}

\begin{abstract}
In this paper we demonstrated the effectiveness of imaging in a tissue phantom with isotropic scattering using polarization discrimination combined with the time gating method. This simple polarization discrimination technique was shown ineffective when it was applied to filamentous tissues. In this situation, we utilized the time-gated degree of polarization (DOP) imaging technique to show that the DOP measurement was quite effective for high-quality imaging of objects in filamentous tissues. We also applied this technique to the characterization of myocardial tissues and showed the difference between normal and abnormal tissues. In addition, we demonstrated a novel method for target depth determination in a turbid medium based on co-polarized light measurements. This method relied on the strong dependence of transmitted co-polarized intensity on target depth.
\end{abstract}

Key words: time gating, turbid medium, polarization gating, optical imaging, tissue characterization, degree of polarization

\section{Introduction}

Ultrafast optics techniques have been widely used for optical imaging of human tissues based on time gating of transmitted photons. In such a technique, short pulses are applied to biological tissues and the transmitted snake photons are extracted with the various time-gating techniques. ${ }^{1-5)}$ Because there is no clear boundary between weakly scattered snake photons and strongly scattered or multiply scattered diffuse photons in the transmitted time-resolved signals, careful design and implementation of the aforementioned time gating techniques are required. While time gating methods have been proved useful for biomedical imaging, polarization discrimination technique was developed to improve the resolution and contrast of images. ${ }^{6-9)}$ This method is based on the depolarization effect in random scattering of tissues. In this effect, the input polarization state is partially preserved in weakly scattered photons. Thus, the quasi-coherent part can be extracted by transmitted copolarized photons.

However, in filamentous tissues the measurement of the depolarization effect by discriminating coherent from incoherent photons becomes less effective when there exists coherent coupling between two perpendicular polarization components. Such coherent coupling phenomena can particularly occur in filamentous tissues with anisotropic structures. ${ }^{10)}$ In this situation, the conventional polarization gating method becomes ineffective in optical imaging. In our research, we have found that by using the time-resolved Stokes-Mueller formalism, images of higher contrast and resolution in filamentous tissues could be achieved. ${ }^{11)}$ Because the Stokes vector provides complete polarization information of transmitted light, this technique can be applied to the monitoring of normal and cardiomyopathic myocardium tissues and their differences compared. ${ }^{12)}$ The $4 \times 4$ full Mueller matrices of myocardium samples were evaluated from the measured Stokes vectors with time-gating

*E-mail address: ccy@cc.ee.ntu.edu.tw processes.

Although the techniques of time gating combined with polarization discrimination can usually provide satisfactory 2-D images, the determination of target depth in a turbid medium is usually quite difficult with trans-illumination measurements. In our study, with the measurement of the transmitted co-polarized intensity, a target depth indicator can be calculated. ${ }^{13)}$ In this target depth determination process, the data of degree of polarization (DOP) can be used as a reference in identifying the overall scattering strength of a system.

In this paper, we review several issues of our research on optical imaging and tissue characterization with polarization discrimination of time-gated signals. More detailed discussion is provided here. The basic principles of time gating combined with polarization discrimination and the results of isotropic phantom scattering are shown in $\$ 2$. Next, the timegated DOP imaging method and its application to myocardial tissue diagnosis are shown in $\$ 3$. Then, a novel method for the determination of target depth in a turbid medium based on the polarized transmitted signal measurements is described in $\S 4$. Finally, conclusions are drawn in $\S 5$.

\section{Time-Gated Image Characteristics in a Phantom of Isotropic Scattering}

Figure 1(a) demonstrates the idea of an ultrafast optical imaging method with an input short pulse. The snake photons, i.e., the leading edge of the transmitted temporal profile, which pass through a turbid medium with fewer scattering events, carry more information of image. However, by including diffuse photons in imaging, strong random scattering blurs the image. The images shown in Fig. 1 were obtained by scanning a sample of diluted milk with a transmission length of $10 \mathrm{~cm} .{ }^{10)}$ Two pieces of lean pork were suspended in the diluted milk as scanning objects. Details of the experiment are described in the next paragraph. As shown in Fig. 1(b), with linearly polarized input signals, the transmitted co-polarized photons are more coherent and 


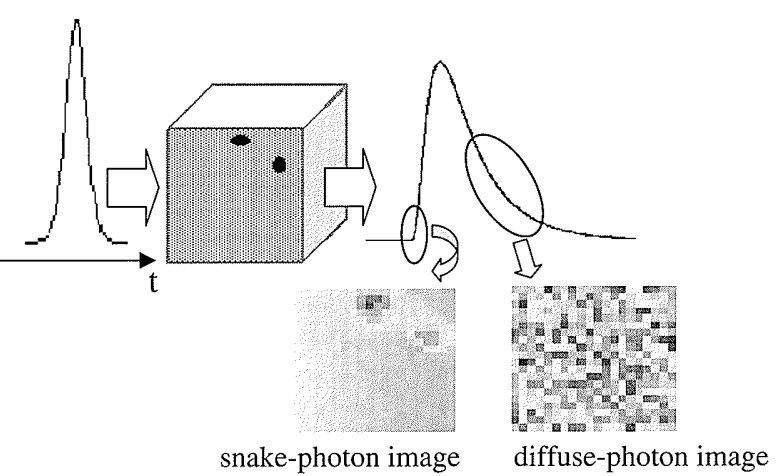

(a)
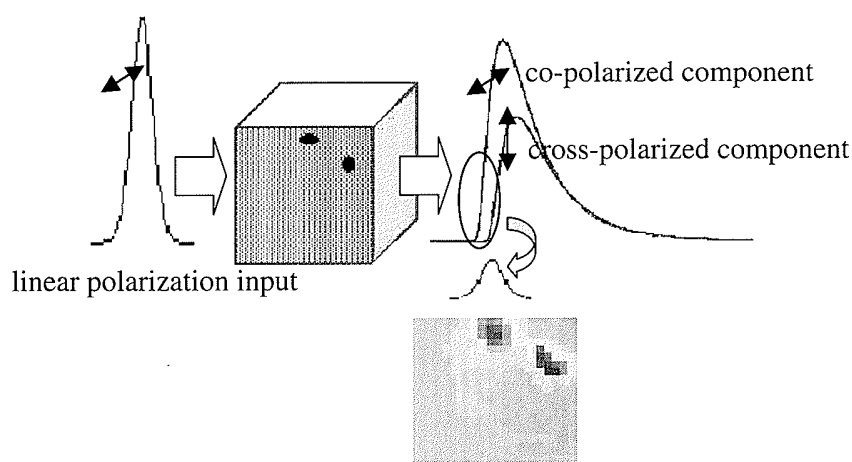

time-gating polarization-discrimination image

(b)

Fig. 1. Illustrations of (a) time-gating and (b) polarization-discrimination combined with time-gating techniques.

carry more information on tissue structures. The subtraction of the cross-polarized component from the co-polarized component, i.e., removal of the incoherent part, has been shown to be a useful procedure for effective optical imaging in a medium of isotropic scattering. In this imaging process, the depolarization factor was usually calibrated from measured data for image construction.

In the experimental setup for optical imaging with polarization-gated snake photons, as shown in Fig. 2, an Argon laser pumped mode-locked Ti:sapphire laser was used to provide $76 \mathrm{MHz}$, around $100 \mathrm{fsec}$ laser pulses at $800 \mathrm{~nm}$. The laser beam was split into three branches, one for triggering the streak camera used, the other for propagating through samples, and the third one for temporal reference. About $100 \mathrm{~mW}$ average power was applied to the samples. The diameter of the laser beam was slightly smaller than $1 \mathrm{~mm}$. Two polarizers, one before and the other after the samples,

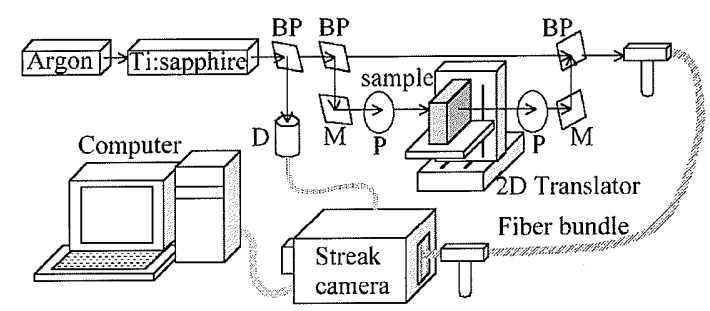

Fig. 2. Experimental setup. BP: beam splitter; P: polarizer; M: mirror; D: detector. were used to control the input and output polarization for polarization discrimination. The sample container was placed on a 2-D translation stage for transverse scanning. After the recombination of the sample and reference beams, signals were directed to the streak camera by a fiber bundle. The effective numerical aperture of the fiber bundle was 0.55 . The streak camera was controlled by commands from a host computer through a GPIB interface. The temporal resolution of the operation mode of the streak camera was about $20 \mathrm{psec}$. This resolution limitation may partly be due to the group-velocity dispersion effect in the fiber bundle. The Ascanning mode was used in measurements, i.e., the fiber bundle end was always aligned with the incident laser beam while the samples were moved with a two-dimensional translation stage for image scanning. The distance between the fiber bundle end and the exiting wall of the sample container was $10 \mathrm{~cm}$ for spatial filtering of snake photons.

As mentioned earlier, two pieces of lean pork ( 2 and $1 \mathrm{~mm}$ in thickness, respectively) as objects were placed within the diluted milk for imaging. The diluted milk was contained in a plastic vessel with the transmission length of $10 \mathrm{~cm}$ as tissue phantom. The probe beam propagated along the $z$-axis with its linear polarization in the horizontal direction, which is defined as the $x$-axis. The scanning pixel size was $1 \times 1 \mathrm{~mm}$ and the scanning area was $2 \times 2 \mathrm{~cm}$. Figure 3 (a) shows the

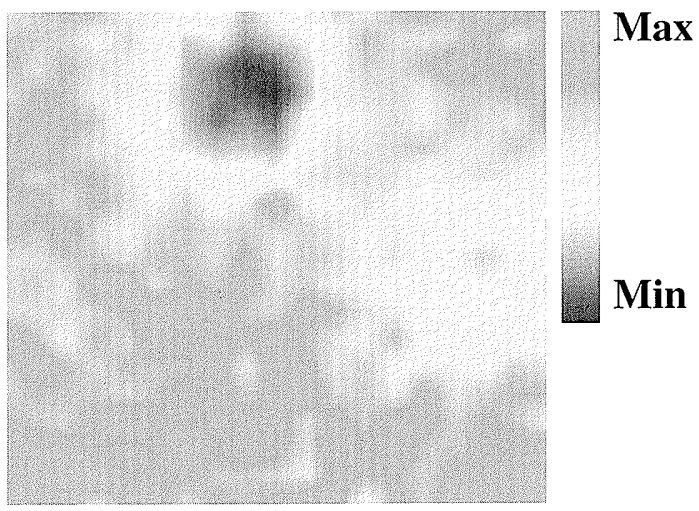

(a)

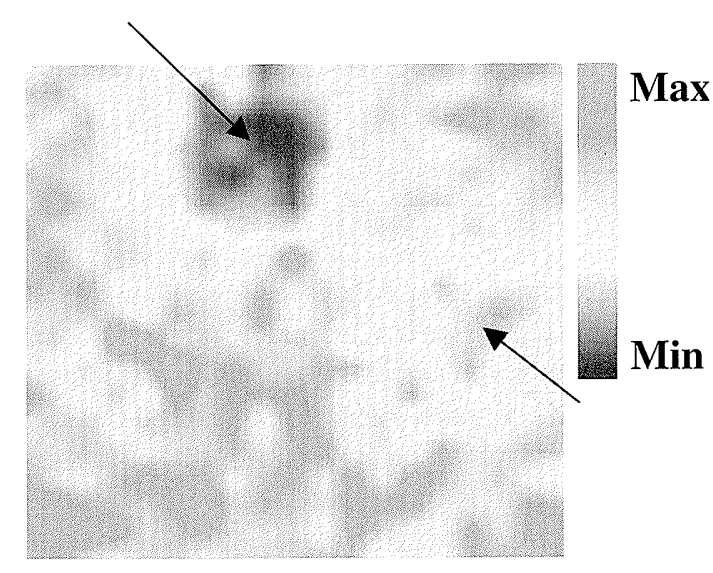

(b)

Fig. 3. Time-gated images of two pieces of lean pork in diluted milk with (a) $I_{x}$ and (b) $I_{x}-I_{y}$. 
image of integrated intensity with appropriate time gating of $I_{x}$. Figure 3(b) shows the image of time-gated $I_{x}-I_{y}$, where $I_{x}$ and $I_{y}$ are co- and cross-polarized components of incident light, respectively. Although $I_{x}$ can show the locations of the two pieces of pork, $I_{x}-I_{y}$ does provide a clearer image, particularly for the smaller and thinner piece on the right. Because diluted milk represents a turbid medium of statistically isotropic scattering, the depolarization effect of transmitted signals is a good indicator of scattering strength. Therefore, the results of polarization discrimination manifest good images in this case.

\section{Time-Resolved DOP Imaging in Filamentous Tissues}

The image results in $\$ 2$ indicate that the combination of polarization-discrimination with the time-gating method improves image contrast in an isotropic tissue phantom. However, as mentioned before, deterministic optical birefringence may result in coherent polarization evolution, leading to coherent polarization coupling and hence invalid coherent photon extraction through polarization discrimination. In this situation, all elements of the polarization state must be considered to understand the polarization evolution and hence improving image quality in such a medium. In this section, we intend to apply the Stokes formalism for improving image contrast in the filamentous tissue. ${ }^{11)}$

Here, the experimental setup is similar to Fig. 2, except that a quarter-wave plate was employed after samples for circularly polarized component measurements. According to the Stokes-Mueller formalism, we need to measure four different polarization states of transmitted photons. The Stokes components, generally denoted by $S_{0}, S_{1}, S_{2}$ and $S_{3}$, form a sufficient set for describing the amplitude, phase and polarization of a light wave. They are defined in terms of the electric fields in the Cartesian coordinate as

$$
S=\left[\begin{array}{c}
S_{0} \\
S_{1} \\
S_{2} \\
S_{3}
\end{array}\right]=\left[\begin{array}{c}
\left|E_{x}\right|^{2}+\left|E_{y}\right|^{2} \\
\left|E_{x}\right|^{2}-\left|E_{y}\right|^{2} \\
2\left|E_{x}\right|\left|E_{y}\right| \cos \delta \\
2\left|E_{x}\right|\left|E_{y}\right| \sin \delta
\end{array}\right] .
$$

Here, $E_{x}$ and $E_{y}$ are the electrical field components in the $x$ and $y$ axes, respectively, and $\delta$ is their relative phase. Meanwhile, the degree of polarization can be evaluated from the Stokes components as

$$
\mathrm{DOP}=\frac{\sqrt{S_{1}^{2}+S_{2}^{2}+S_{3}^{2}}}{S_{0}}, \quad 0 \leq \mathrm{DOP} \leq 1 .
$$

In the experiments, such polarization control was accomplished by adjusting a polarizer and a quarter-wave plate. Here, the horizontal and vertical polarization directions refer to the coordinate of the laboratory. The quasi-coherent photon data were obtained by gating the time-resolved intensity profiles with a duration of $50 \mathrm{psec}$ from the leading edges of the temporal curves. We used chicken breast tissues $1.5 \mathrm{~cm}$ thick as filamentous tissue samples in the experiments.

To demonstrate the improvement of image contrast based on the time-gated Stokes vector, we stuck a thin chicken bone

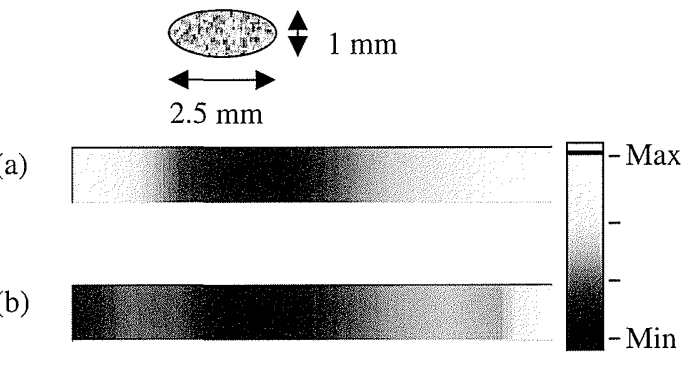

(c)

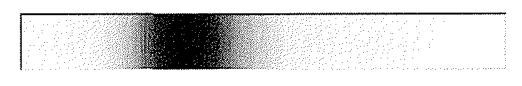

(d)

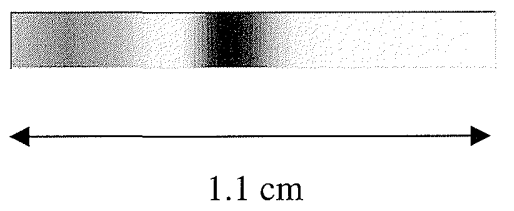

Fig. 4. 1-D images of the chicken bone in chicken breast tissue with filament orientation in the horizontal direction: (a) time-gated integrated intensity $\left(S_{0}\right)$, (b) time-gated $S_{1}$ component, (c) DOP with time gating, and (d) DOP without time gating.

(its shape and size are shown in Fig. 4) into the chicken breast tissue, with bone elongation in the vertical polarization direction for optical imaging. Line scan images were obtained by linear scanning in the direction perpendicular to the bone elongation, i.e., scanning across the bone. The spatial range in the abscissa is $1.1 \mathrm{~cm}$ with the step size of $1 \mathrm{~mm}$. The gray level represents the relative intensity distribution. In Fig. 4, we compare various gating schemes, including (a) time gated integrated intensity $\left(S_{0}\right)$, (b) timegated $S_{1}$ component, i.e., the conventional approach of combining polarization discrimination with time gating, (c) DOP distribution with time gating, and (d) DOP distribution without time gating. As shown in part (d), even a rough image cannot be obtained without time gating. The comparison between parts (a)-(c) shows that although images of the chicken bone were obtained with time gating and/or polarization discrimination, an image of higher resolution and contrast was achieved with DOP evaluation. The variation of gray level in the bone region indicates the gradient of bone thickness. This result implies that the time-gated DOP measurement can substitute for the conventional polarization-discrimination method in filamentous tissues for optical imaging.

According to the time-gated Stokes-Mueller measurement, we also prepared myocardial tissues of hamsters for optical diagnosis. ${ }^{12)}$ The Mueller matrices of normal and cardiomyopathic myocardium evaluated from the measured Stokes vectors are shown in Figs. 5(a) and 5(b), respectively. The time-gated result was obtained by integration of the timeresolved intensity profiles with duration of about $50 \mathrm{ps}$ from the leading edges of the profiles. The 3-D mapping image represents a $4 \times 4$ full Mueller matrix. The quantities of diagonal elements in the Mueller matrices stand for the intensities of preserved original polarization states from various polarization inputs. The quantities of diagonal 


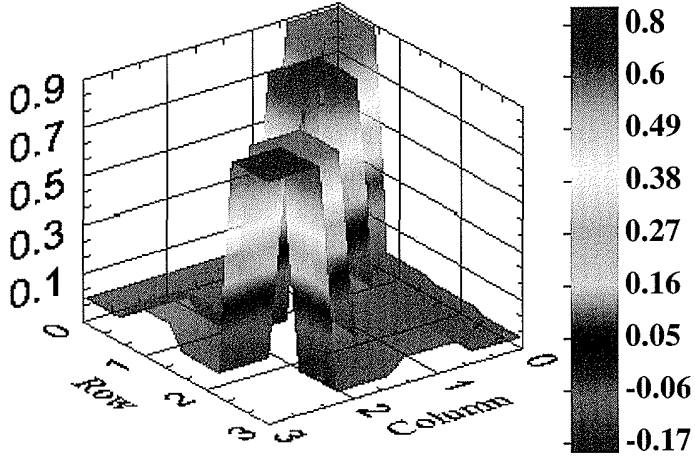

(a)

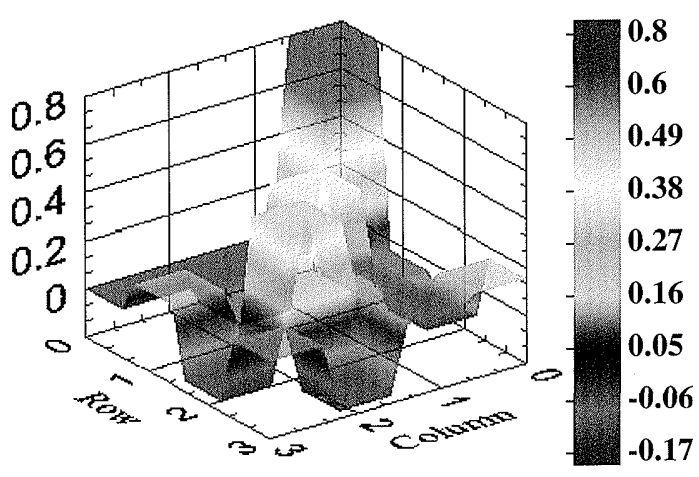

(b)

Fig. 5. Time-resolved Mueller matrices of (a) normal and (b) cardiomyopathic myocardium.

elements in Fig. 5(b) are lower than those of Fig. 5(a) which reveals the stronger scattering in the damaged fiber architecture. Meanwhile, the pattern in Fig. 5(b) manifests the difference of polarization evolution from that of Fig. 5(a) due to the histological alterations of the fibrous structure in cardiomyopathic myocardium. In our experiments, the cardiomyopathic myocardium sample (about $6 \mathrm{~mm}$ in thickness) is significantly thicker than the normal sample (about $4 \mathrm{~mm}$ ). Such thickness difference is believed due to the cardiomyopathic effect in the tissue. The temporal profiles of the measurement with the cardiomyopathic myocardium sample were significantly longer than those of the normal myocardium. Such elongation can be attributed to the thickened sample and the enlargement of scattering coefficient of the abnormal sample. However, it is usually difficult to observe in vivo such alterations of tissue structure. Therefore, more information like Mueller matrix is needed for myocardial tissue characterization. The Stokes-Mueller measurements that we have presented here is a promising method for such a tissue diagnosis.

\section{Determination of Target Depth in a Phantom with Polarization Discriminated Transmitted Signals}

In this part, the experimental setup is again similar to Fig. 2. ${ }^{13)}$ The geometry of the cubic container of microsphere suspension was $5 \times 5 \times 2.5 \mathrm{~cm}$ (signal transmission length $2.5 \mathrm{~cm}$ ). The A-scan mode was used in measurements. The 1-D scanning step size was $1 \mathrm{~mm}$ and the scanning length was $1.8 \mathrm{~cm}$. In sample preparation, white polystyrene latex was diluted with water to serve as the turbid background medium. The average diameter of polystyrene micro-spheres (refractive index 1.565) was $1.8 \mu \mathrm{m}$. The volume concentration of diluted polystyrene suspension was $0.067 \%$. The inhomogeneous target was jellied $0.134 \%$ polystyrene suspension $4 \mathrm{~mm}$ in thickness. It had a sharp boundary at the origin of the transverse coordinate $y=0$.

Figure 6(a) shows the experimental results of the transmitted co-polarized intensities as functions of the transverse scanning position, $y$, for four different target depths, $x$. These intensities were obtained by integration over the whole range of the time-resolved co-polarized intensity profiles. The curves show the results of transverse scanning across the boundary of the target at $y=0$. Abrupt changes of transmitted co-polarized intensity across the target boundary can be clearly seen. Although the transmitted intensity on the target-free side is almost constant in varying the target depth, that on the target side increases significantly with target

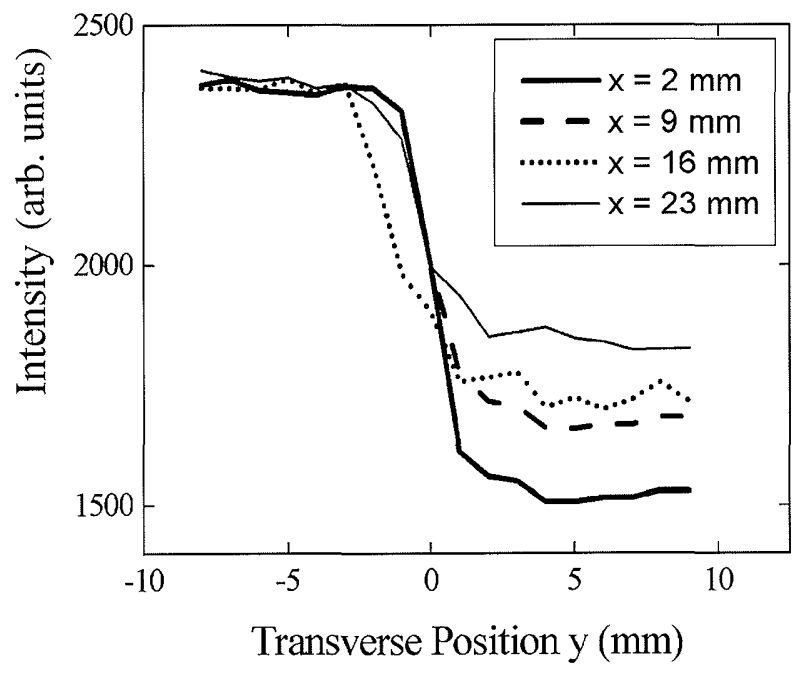

(a)

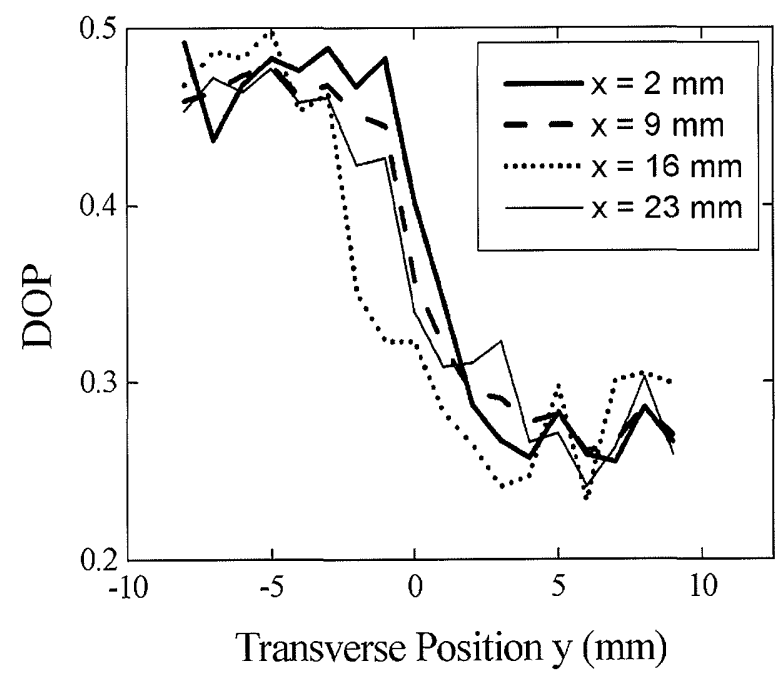

(b)

Fig. 6. 1-D images of (a) the transmitted co-polarized intensity and (b) the DOP of four target depths obtained from the experiments. 
depth. Such variations can be explained as follows. When the target is closer to the transmitter, the scattered photons travel a longer distance after the influence of the target of stronger scattering. In this situation, photons of random propagation directions evolve into a weaker intensity at the receiver of a finite aperture, particularly in the co-polarized component. With this dependence of transmitted intensity, the depth information can be extracted.

Figure 6(b) shows the 1-D scan images of DOP under the same conditions as those of Fig. 6(a). Again, these results were obtained by integrating over the whole range of the time-resolved intensity profiles. The DOP values drop from about 0.48 to around 0.27 when we scan across the target boundary. Almost the same DOP value on the target side in varying the target depth represents a major difference from the results of co-polarized intensity. Such a difference is due to the fact that the DOP value, controlled by ballistic and snake photons, is mainly determined by the total length of nearly straight photon paths, which is less influenced by the target depth. This method of depth determination basically has no limitation in application. However, if the geometry of the target becomes complicated, the data processing procedures can be time consuming. With Monte Carlo simulations, we have shown that with the target and background medium absorption coefficients up to $0.3 \mathrm{~cm}^{-1}$, the target depth determination method still works. In the current research, we assume that the scatterings of the target and background media are isotropic. In the cases of media with anisotropic random scattering or deterministic birefringence nature, such a target depth determination method is theoretically still useful. However, its implementation requires further investigation.

\section{Conclusions}

We first demonstrated the effectiveness of imaging in a phantom with isotropic scattering using polarization discrimination combined with time gating. However, in filamentous tissues this conventional imaging method became less effective because filamentous tissues had a deterministically anisotropic property. In this situation, we employed the timegated DOP imaging technique and found that it was quite effective in high-quality imaging of objects in filamentous tissues. This is so because the Stokes vector provides complete polarization information of transmitted signals. Meanwhile, application of this technique to the diagnosis of myocardial tissues of hamsters was proved useful. Then, we developed a novel method for target depth determination in a turbid medium. This method relied on the strong dependence of transmitted co-polarized intensity on target depth. A target of stronger scattering located closer to the transmitter resulted in stronger photon divergence and hence weaker co-polarized intensity at the receiver.

\section{Acknowledgement}

This research was supported by the National Health Research Institute of The Republic of China, under the grant NHRI-EX92-9220EI.

\section{References}

1) L. Wang, P. P. Ho, C. Liu and R. R. Alfano: Science 253 (1993) 769.

2) R. R. Alfano, X. Liang, L. Wang and P. P. Ho: Science 264 (1994) 1913.

3) S. K. Gayen and R. R. Alfano: Opt. Express 4 (1999) 475.

4) D. Grosenick, H. Wabnitz, H. H. Rinneberg, K. T. Moesta and P. M. Schlag: Appl. Opt. 38 (1999) 2927.

5) S. K. Gayen, M. Alrubaiee, H. E. Savage, S. P. Schantz and R. R. Alfano: IEEE J. Sel. Top. Quant. Elec. 7 (2001) 906.

6) H. Horinaka, K. Hashimoto, K. Wada, Y. Cho and M. Osawa: Opt. Lett. 20 (1995) 1501.

7) S. G. Demos and R. R. Alfano: Opt. Lett. 21 (1996) 161.

8) X. Gan, S. P. Schilders and Min Gu: J. Opt. Soc. Am. A 16 (1999) 2177.

9) V. Sankaran, K. Schönenberger, J. T. Walsh, Jr. and D. J. Maitland: Appl. Opt. 38 (1999) 4252

10) C.-W. Sun, C.-Y. Wang, C. C. Yang, Y.-W. Kiang, I.-J. Hsu and C.-W. Lin: Opt. Lett. 26 (2001) 432.

11) C.-W. Sun, C. C. Yang and Y.-W. Kiang: Appl. Opt. 42 (2003) 750.

12) C.-W. Sun, L.-S. Lu, C. C. Yang, Y.-W. Kiang and M.-J. Su: Opt. Express 10 (2002) 1347.

13) H.-S. Wang, C.-W. Sun, Y.-W. Kiang and C. C. Yang: Opt. Lett. 28 (2003) 25 . 